\section{INPLASY PROTOCOL}

To cite: Lin et al. Retinal nerve fiber layer changes in migraine: a protocol for systematic review and meta analysis. Inplasy protocol 202060033. doi:

10.37766/inplasy2020.6.0033

Received: 10 June 2020

Published: 10 June 2020

Corresponding author: PanWen Zhao

zhaopanwen8@163.com

Author Affiliation:

Affiliated Yancheng Hospital, School of Medicine

\section{Support: NSFC 81601161}

Review Stage at time of this submission: Piloting of the study selection process.

\section{Conflicts of interest:}

The authors have no conflicts of interest to disclose.

\title{
Retinal nerve fiber layer changes in migraine: a protocol for systematic review and meta analysis
}

Lin, XG1; Yi, ZQ2; Zhang, XL3; Liu, QQ4; Cai, RY5; Chen, CC6; Zhang, $\mathrm{HJ}^{7}$; Zhao, PW8; Pan, PL9.

Review question / Objective: The purpose of the study was to assess the relationship between migraine and changes in retinal nerve fiber layer thickness measured using optical coherence tomography.

Condition being studied: Migraine is one of the most disability-causing diseases worldwide, affected about $14 \%$ of the global population. Optical coherence tomography (OCT) is a common non-invasive imaging technique for measuring the retina and retinal nerve fiber layer (RNFL). A number of studies have used OCT to investigate RNFL alterations in migraine; however, the results were not consistent and even conflicting. Some studies have found that the thickness of the RNFL in patients with migraine has decreased, while others have observed increased thickness. An earlier meta-analysis published in 2015 (DOI: 10.3109/02713683.2015.1056373) evaluated RNFL thickness measurements with OCT in migraine patients. However, this meta-analysis included only 6 studies. Since then, many studies using SD-OCT have been published. Previous studies have shown that RNFL thickness is affected by the type of OCT device used. Although both time-domain (TD) OCT and spectral-domain (SD) OCT studies were included in the meta-analysis, there was no subgroup analysis of the instrument. Therefore, we will make an update on this meta-analysis and further evaluate the effect of different OCT instruments.

INPLASY registration number: This protocol was registered with the International Platform of Registered Systematic Review and Meta-Analysis Protocols (INPLASY) on 10 June 2020 and was last updated on 10 June 2020 (registration number INPLASY202060033).

\section{INTRODUCTION}

Review question / Objective: The purpose of the study was to assess the relationship between migraine and changes in retinal nerve fiber layer thickness measured using optical coherence tomography. 
Condition being studied: Migraine is one of the most disability-causing diseases worldwide, affected about $14 \%$ of the global population. Optical coherence tomography (OCT) is a common noninvasive imaging technique for measuring the retina and retinal nerve fiber layer (RNFL). A number of studies have used OCT to investigate RNFL alterations in migraine; however, the results were not consistent and even conflicting. Some studies have found that the thickness of the RNFL in patients with migraine has decreased, while others have observed increased thickness. An earlier metaanalysis published in 2015 (DOI: 10.3109/02713683.2015.1056373) evaluated RNFL thickness measurements with OCT in migraine patients. However, this metaanalysis included only 6 studies. Since then, many studies using SD-OCT have been published. Previous studies have shown that RNFL thickness is affected by the type of OCT device used. Although both time-domain (TD) OCT and spectraldomain (SD) OCT studies were included in the meta-analysis, there was no subgroup analysis of the instrument. Therefore, we will make an update on this meta-analysis and further evaluate the effect of different OCT instruments.

\section{METHODS}

Search strategy: We searched the electronic databases PubMed, EMBASE and Web of Science. The primary search strategies were: ((Optical Coherence Tomography) or (Retina Nerve Fiber Layer) or RNFL) and Migraine. The date of the last search is set at $18 \mathrm{March}, 2020$. Additionally, the reference lists of relevant reviews and the articles selected for inclusion were manually searched.

Participant or population: Inclusion criteria: - Patients diagnosed with migraine by International Headache Disease Classification. - Normal visual field. Adults (over the age of 18). Exclusion criteria: - Incorporate any form of glaucoma, optic nerve disease, or intraocular surgical intervention. - Diabetes with any sign of retinopathy (hemorrhages, hard and/or soft exudates, macular edema). - Neurological disorders that may affect RNFL thickness. - Children (under 18 years of age).

Intervention: The main subject (intervention/exposure) of the study is to use optical coherence tomography (OCT) to evaluate the effects of migraine on the thickness of the mean and segmental retinal nerve fiber layer (RNFL).

Comparator: Healthy controls.

Study designs to be included: This study selected only case-control studies using optical coherence tomography to measure the thickness of the retinal nerve fiber layer in migraine.

Eligibility criteria: Inclusion criteria: - Casecontrol studies. - Patients with migraine were compared with healthy controls. - All studies used OCT measurements and reported retinal nerve fiber layer thickness. - All articles in English. Exclusion criteria: Animal studies, abstracts, letters, reviews non-English studies and case-studies. The outcome values cannot be used for meta-analysis.

Information sources: The search will be performed in PubMed, EMBASE and Web of Science with a language limitation of English. We will retrieve each database from the built-in until March 27, 2020.

Main outcome(s): Differences in mean RNFL thickness and segmental RNFL thickness in migraine patients and health controls.

Additional outcome(s): None.

Data management: We will ensure the robustness of the results through repeated analysis using different effect size indicators (MD, SMD, etc.) and different statistical models (fixed effect and random effect models).

Quality assessment / Risk of bias analysis: We will use the Newcastle-Ottawa Scale (NOS) to evaluate the quality of case- 
control studies included in this metaanalysis. This scale includes three aspects of evaluation: case group and control group selection methods, comparability between case groups and control groups, and exposure assessment.

Strategy of data synthesis: For metaanalysis we will use Stata 15. Heterogeneity will be assessed by $\left.\right|^{2}$ test among included studies. If $I^{2}<50 \%$, the data are synthesized using a fixed effect model. Otherwise, a random effect model is performed, and sensitivity analysis and subgroup analysis will be conducted to explain the source of heterogeneity.

Subgroup analysis: Type of migraine; Segmental RNFL; Spectral-domain (SD) OCT vs. time domain (TD) OCT.

Sensibility analysis: In order to investigate the stability of the results, we performed sensitivity analysis, excluding studies with low methodological quality one by one, to investigate the effect of individual studies on the overall results, and whether these results were reliable.

Language: English.

Country(ies) involved: China.

Keywords: Migraine; Retinal nerve fiber layer; Optical coherence tomography; Migraine without aura; Migraine with aura.

Contributions of each author:

Author 1 - XiaoGuang Lin - The author drafted the manuscript.

Author 2 - ZhongQuan Yi - The author contributed to data analysis and provided statistical expertise.

Author 3 - XueLing Zhang - The author contributed to the development of the selection criteria, the risk of bias assessment strategy, data extraction, and drafted the manuscript.

Author 4 - QinQin Liu - The author contributed to the development of the selection criteria, the risk of bias assessment strategy, data extraction, and drafted the manuscript.
Author 5 - RuYuan Cai - The author provided methodological support and software analysis guidance.

Author 6 - ChaoChun Chen - The author provided methodological support and software analysis guidance.

Author 7 - HongJie Zhang - The author provided methodological support and software analysis guidance.

Author 8 - PanWen Zhao - The author contributed to conception, design, data analysis, and writing the manuscript.

Author 9 - PingLei Pan - The author contributed to the editing and revision of the manuscript. 